\title{
Canonical polyadic decomposition for tissue type differentiation using multi-parametric MRI in high-grade gliomas
}

\author{
H. N. Bharath ${ }^{1,2}$, N. Sauwen ${ }^{1,2}$, D. M. Sima ${ }^{1,2}$, U. Himmelreich ${ }^{3}$, L. De Lathauwer ${ }^{1,4}$, S. Van Huffel ${ }^{1,2}$
}

\begin{abstract}
In diagnosis and treatment planning of brain tumors, characterisation and localization of tissue plays an important role. Blind source separation techniques are generally employed to extract the tissue-specific profiles and its corresponding distribution from the multi-parametric MRI. A 3-dimensional tensor is constructed from in-vivo multiparametric MRI of high grade glioma patients. Constrained canonical polyadic decomposition (CPD) with common factor in mode- 1 and mode- 2 and $l_{1}$ regularization on mode- 3 is applied on the 3-dimensional multi-parametric tensor to characterize various tissue types. An initial in-vivo study shows that CPD has slightly better performance in identifying active tumor and the tumor core region in high-grade glioma patients compared to hierarchical non-negative matrix factorization.
\end{abstract}

\section{INTRODUCTION}

Accurate characterisation and localization of tissue types play a key role in brain tumor diagnosis and treatment planning. Neuro-imaging methods provide anatomical and pathophysiological information about brain tumors and aid in diagnosis, treatment planning and follow-up of patients. Currently, conventional magnetic resonance imaging (cMRI) is mainly used for detection and analysis of brain tumor upon suspicion. In recent years, many advanced magnetic resonance (MR) modalities, such as perfusion-weighted imaging (PWI), diffusion-weighted imaging (DWI) and MR spectroscopic imaging (MRSI) are being used for brain tumor diagnosis [1], [2], [3]. Instead of using the MR modalities independently, it has been shown that combining several MRI modalities (multi-parametric MRI) improves diagnostic accuracy when it comes to grading gliomas [4], [5]. The tumor region of glioblastoma multiforme (GBM) could consist of several tissue types, which represent actively growing tumor, necrosis or normal brain tissue [1]. Hierarchical nonnegative matrix factorization (hNMF) has been applied to brain MRSI data to differentiate various tissue patterns in GBM patients [1]. Recently, a variation of hierarchical nonnegative matrix factorization (hNMF) algorithm was applied to multi-parametric MRI (MP-MRI) data for tumor tissue segmentation [5].

Tensorizing the matrix and using suitable tensor decompositions has certain advantages in blind source separation

\footnotetext{
${ }^{1}$ Department of Electrical Engineering (ESAT), STADIUS Center for Dynamical Systems, Signal Processing and Data Analytics, KU Leuven, Leuven, Belgium.

${ }^{2}$ iMinds Medical Information Technologies, Leuven, Belgium.

${ }^{3}$ Biomedical MRI Unit/Molecular Small Animal Imaging Center, Department of Imaging and Pathology, KU Leuven, Leuven, Belgium.

${ }^{4}$ The Group of Science, Engineering and Technology, KU Leuven Kulak, Kortrijk, Belgium.
}

problems [6]. In [7] non-negative canonical polyadic decomposition has been used for tissue differentiation from MRSI data. In this paper, a tensor based algorithm is proposed for tissue type differentiation in high-grade glioma patients from MP-MRI. A modified version of the tensor approach in [7] has been applied to MP-MRI imaging. The proposed method consists of representing the MP-MRI data in a 3-dimensional tensor and applying constrained canonical polyadic decomposition (CPD) with $l_{1}$ regularization to the MP-MRI tensor. The performance of the CPD algorithm was evaluated using expert segmentation and compared with hierarchical nonnegative matrix factorization (hNMF) [5].

\section{MATERIALS}

\section{A. Data acquisition}

All the MP-MRI acquisition was performed on a 3T MR system (Philips Achieva, The Netherlands) at the University Hospital of Leuven (UZ Leuven), using a body coil for transmission and an 8-channel head coil for signal reception. The total scanning time for all MRI modalities was around 45 min. 14 high-grade glioma patients (11 grade IV, 2 grade III and one grade II astrocytoma with focal progression to a grade III glioma) were scanned for this study. The lesions were classified according to grade using the 2007 WHO classification [8], with histopathological confirmation in all cases. The study involving human subjects has been approved by the human ethics review board of the institute. Written informed consent was obtained from every patient before participation.

\section{B. Conventional magnetic resonance imaging (cMRI)}

An axial spin echo T2-weighted MRI was acquired with the following parameters: repetition time (TR)/ echo time (TE): 3000/80 ms; slice/gap: $4 / 1 \mathrm{~mm}$; turbo factor: 10 ; field of view [FOV]: $230 \times 184 \mathrm{~mm}^{2}$; acquisition matrix: $400 \times 300$.

A T1-weighted 3-D spoiled gradient echo MRI scan with contrast administration was performed with the following parameters: fast field echo, TR/TE/inversion time (TI): 9.7/4.6/900 ms; flip angle: $8^{\circ}$; turbo field echo factor: 180 ; acquisition voxel size: $0.98 \times 0.98 \times 1 \mathrm{~mm}^{3} ; 118$ contiguous partitions.

An axial FLAIR MRI scan was acquired with the following parameters: TR/TE/TI: 11000/120/2800 ms, slice/gap: 4/1 mm, FOV: $230 \times 184 \mathrm{~mm}^{2}$, acquisition matrix: $240 \times 134$. 


\section{Perfusion weighted imaging (PWI)}

Perfusion images were obtained using Dynamic Susceptibility-weighted Contrast-enhanced (DSC) MRI with a gradient-echo EPI sequence: TR/TE: 1350/30 ms; slice/gap: 3/0 mm; dynamic scans: 60; FOV: $200 \times 200$ $\mathrm{mm}^{2}$; matrix: $112 \times 109$; scan time: around $1 \mathrm{~min} 26 \mathrm{~s}$. EPI data were acquired during the first pass following a rapid injection of a $0.1 \mathrm{mmol} / \mathrm{kg}$ body weight bolus of meglumine gadoterate (Dotarem, Guerbet, France) via a mechanical pump at a rate of $4 \mathrm{~mL} / \mathrm{s}$, followed by a $20-\mathrm{mL}$ bolus of saline.

DSC data were analyzed using the DSCoMAN plugin [9] in ImageJ [10]. Relative cerebral blood volume (rCBV) maps were derived from the dynamic signal intensity curves using the method proposed by Boxerman et al. [11].

\section{Magnetic resonance spectroscopic imaging (MRSI)}

A 2D-1H MRSI protocol was used as previously described in [12]. A point-resolved spectroscopy sequence was used as the volume selection technique with a bandwidth of $1.3 \mathrm{kHz}$ for the conventional slice-selective pulses; TR/TE: 2000/35ms; FOV: $160 \times 160 \mathrm{~mm}^{2}$; maximal volume of interest (VOI): $80 \times 80 \mathrm{~mm}^{2}$; slice thickness: $10 \mathrm{~mm}$; acquisition voxel size: $10 \times 10 \mathrm{~mm}^{2}$; reconstruction voxel size: $5 \times 5 \mathrm{~mm}^{2}$; receiver bandwidth: $2000 \mathrm{~Hz}$; samples: 2048; number of signal averages: 1; water suppression method: multiple optimizations insensitive suppression train [13]; first- and secondorder pencil beam shimming; parallel imaging: sensitivity encoding with reduction factors of 2 (left-right) and 1.8 (anterior-posterior); scan time: $3 \mathrm{~min} 30 \mathrm{~s}$.

The raw MRSI data were exported from the Philips platform after standard post-processing (zero-filling in kspace, transformation from k-space to spatial domain, automatic phase correction and eddy current correction). Further processing was done with MatLab 2012b (MathWorks, Massachussets, USA) based SPID software [14]. AQSES-MRSI [15] was used to quantify the following metabolites using an experimentally acquired metabolite basis set: Lip at $1.3 \mathrm{ppm}$, Lac, NAA, Glx, Cre, Cho, mI and Gly. The residual water was removed using MPFIR (Maximum-Phase Finite Impulse Response) filtering [16], a model order 25 and the spectral range from 0.25 to $4.2 \mathrm{ppm}$ was used. A band of voxels at the outer edges of the VOI was omitted to avoid chemical shift displacement artifacts and lipid contamination.

\section{E. Diffusion kurtosis imaging (DKI)}

An EPI DWI sequence with a spin-echo readout was used to acquire the DKI data, according to an optimized DKI protocol [17]. Implemented b-values were 0, 700, 1000, and $2800 \mathrm{~s} / \mathrm{mm}^{2}$, applied in respectively $10,25,40$, and 75 uniformly distributed directions. The following parameters were used throughout the DKI acquisition sequence: TR/TE: $3200 / 90 \mathrm{~ms}$; gradient duration/diffusion time interval: 20/48.3 ms; FOV: $240 \times 240 \mathrm{~mm}^{2}$; matrix: $96 \times 96$; number of signal averages: 1 ; slice/gap: $2.5 / 0 \mathrm{~mm}$; parallel imaging: SENSE with factor 2 in the antero-posterior direction. The scan time was $17 \mathrm{~min} 30 \mathrm{~s}$.
After motion and eddy current correction [18], isotropic smoothing was applied using a Gaussian kernel (FWHM $=3 \mathrm{~mm})$ to reduce Gibbs ringing. However, smoothing was applied to the $b=0$ and $b=700$ images only, so as not to alter the nature of the noise distribution in the highly diffusion-weighted images in order to avoid a noise bias in the subsequent tensor estimation [19]. Diffusion and kurtosis tensors were estimated in each voxel using a constrained weighted linear least-squares algorithm [19]. Mean diffusivity (MD), fractional anisotropy (FA) and mean kurtosis (MK) maps were derived from the tensors according to [17], [20].

\section{F. Data co-registration}

All the MP-MRI modalities were co-registered and brought to the same spatial resolution to perform voxel-wise analysis. Cubic spline interpolation was used to create an interpolated set of $\mathrm{T} 1+\mathrm{C}$ images, with one slice coinciding with the central plane of the MRSI volume of interest. Only the MRI data that are within the MRSI region of interest (ROI) were considered for analysis. Skull-stripping was applied to all images prior to co-registration. The cMRI datasets and the PWI dataset were rigidly co-registered to the interpolated $\mathrm{T} 1+\mathrm{C}$ reference set using SPM [21]. The normalized mutual information criterion was used for coregistration [22], with cubic spline interpolation for reslicing. Diffusion-weighted parameter maps were non-linearly coregistered to the T2-weighted MRI (co-registered with $\mathrm{T} 1+\mathrm{C}$ ) using ExploreDTI [23] to minimize the local misalignment between the EPI distorted DKI data and the cMRI data. The MRSI data were spatially aligned with the reference set and resampled using cubic spline interpolation. All MRI parameters were brought to the spatial resolution of the original $\mathrm{T} 1+\mathrm{C}$ dataset, i.e. $0.98 \times 0.98 \times 1 \mathrm{~mm}^{3}$. Only the voxels that are within the MRSI ROI are considered for analysis.

\section{MethoD}

\section{A. Tensor construction}

For each voxel in the ROI, a vector $X$ is constructed consisting of MP-MRI features as shown in Fig. 1a. The vector $\mathrm{X}$ consists of:

1) 7 metabolite concentrations mI, Gly, Cho, Cre, Glx, NAA, Lac and Lip from the MRSI modality.

2) $\mathrm{T} 2, \mathrm{~T} 1+\mathrm{C}$ and FLAIR from the cMRI modality.

3) rCBV values from the PWI modality.

4) $\mathrm{MD}, \mathrm{MK}$ and FA values from the DKI modality.

5) Smoothed version of T2, T1+C, FLAIR, rCBV, MD, MK and FA features using a moving average window with kernel size $3 \times 3$.

6) Smoothed version $\mathrm{T} 2, \mathrm{~T} 1+\mathrm{C}$, FLAIR, rCBV, MD, MK and FA features using a moving average window with kernel size $5 \times 5$.

A 3-way MP-MRI tensor $\mathcal{T}$ is constructed by stacking $X X^{T}$ from all the voxels in the MP-MRI grid as shown in Fig. 1b. 
(a)

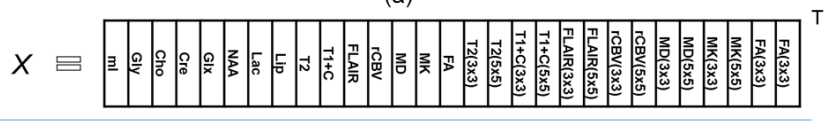

(b)

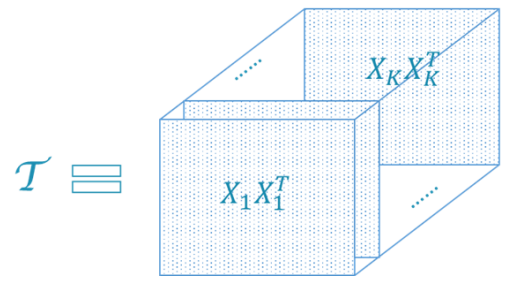

Fig. 1: (a) Construction of feature vector $X$ from MP-MRI. (b) Construction of the MP-MRI tensor $\mathcal{T}$ from the feature vector $X . K$ is the total number of voxels in the region of interest (ROI).

\section{B. Constrained canonical polyadic decomposition}

Canonical polyadic decomposition (CPD) is a tensor decomposition method, where the tensor is decomposed into a sum of rank-one tensors [24]. CPD of a third-order tensor $x \in \mathbb{R}^{I \times J \times K}$ can be written as

$$
\chi \approx \llbracket A B C \rrbracket \equiv \sum_{r=1}^{R} a_{r} \circ b_{r} \circ c_{r},
$$

where $A=\left[a_{1}, a_{2}, \ldots, a_{R}\right] \in \mathbb{R}^{I \times R}, B=\left[b_{1}, b_{2}, \ldots, b_{R}\right] \in \mathbb{R}^{J \times R}$ and $C=\left[c_{1}, c_{2}, \ldots, c_{R}\right] \in \mathbb{R}^{K \times R}$ are factor matrices. $R$ is the rank, defined as the number of rank-one terms. ' $\circ$ ' represents outer product.

In the MP-MRI tensor $\mathcal{T}$, the frontal slices are symmetrical, therefore we constrain the frontal slices of each CPD rank-one term to be symmetric. To maintain symmetry, a common factor matrix is used for mode- 1 and mode- 2 in the CPD as shown in Fig. 2. Tissue abundances are kept positive by imposing a non-negative constraint on the mode-3 factor matrix in the CPD.

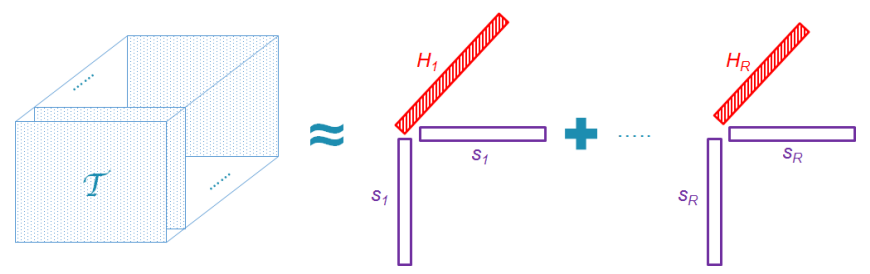

Fig. 2: Partial non-negative CPD of MP-MRI tensor $\mathcal{T}$ : MPMRI tensor $\mathcal{T}$ is decomposed into $R$ rank-1 tensors. Common factor $S$ is used in mode- 1 and mode- 2 to maintain symmetry of frontal slices. Each $s_{i}$ gives a tissue-specific feature vector and the corresponding $h_{i}$ gives the spatial distribution of the respective tissue type, upon reshaping. A non-negativity constraint is imposed only on $H$ in the decomposition.

Each rank-one term obtained from the constrained CPD of the MP-MRI tensor $\mathcal{T}$ is expected to correspond to a particular tissue type. Therefore, the mode- 3 factor matrix, $H$ will be sparse, meaning that each row will mostly have only one high value. $l_{1}$ regularization is applied on the mode- 3 factor matrix $H$ in the partial non-negative CPD to exploit the sparsity assumption of the tissue type distribution.

$$
\left[S^{*}, H^{*}\right]=\arg \min _{S, H \geq 0}\left\|\mathcal{T}-\sum_{i=1}^{R} S(:, i) \circ S(:, i) \circ H(:, i)\right\|_{2}^{2}+\lambda\|V e c(H)\|_{1},
$$

where $S$ represents a matrix with tissue-specific feature vectors as columns, $H$ is a matrix containing their corresponding distribution vectors and $\lambda$ is the parameter which controls the sparsity. The tensor decomposition was performed using Tensorlab Matlab package [25]. Common mode-1 and mode2 factor, non-negativity of $H$ (mode- 3 ) and $l_{1}$ regularization are applied using structured data fusion method [26] in Tensorlab.

\section{Validation}

Dice score: For each patient, the tissue segmentation were obtained from their corresponding abundance values, $H$. The tissue segmentation was performed by assigning each voxel to a particular source using k-means clustering on the abundance values, $H$. Number of classes is set equal to $R$, the number of sources obtained from CPD. The segmentation obtained from CPD was compared to the manual segmentation by a radiologist (SVC, with 6 years of experience in brain tumor research) using the dice score:

$$
\text { Dice }_{\text {tissue }}=2 \times \frac{A_{\text {tissue }, \text { CPD }} \cap A_{\text {tissue,expert }}}{A_{\text {tissue }, C P D}+A_{\text {tissue, expert }}}
$$

where, Dice $_{\text {tissue }}$ is the dice score, $A_{\text {tissue }, C P D}$ is the area of tissue segmentation obtained from the CPD algorithm and $A_{\text {tissue,expert }}$ is the tissue area labelled by a radiologist.

Source correlation: In this method Pearson's linear correlation coefficient is calculated between the estimated feature vectors and the tissue-specific feature vectors based on expert labelling of the in-vivo MP-MRI voxels [1]. The expert label based feature vector for a particular tissue type is computed as the average of all the feature vectors from the voxels which are labelled by the expert as belonging to that tissue type.

\section{EXPERIMENTAL RESULTS}

The feasibility of constrained CPD algorithm in differentiating tissue types is tested by applying it on 14 MPMRI datasets from patients with high-grade glioma. The performance of the constrained CPD algorithm is compared with the hNMF algorithm [5]. Table I shows the dice score for active tumor and the tumor core region and source correlation for the active tumor tissue type as computed by the constrained CPD and the hNMF algorithm. Table I also shows the mean and standard deviation of the dice score and source correlation over 14 MP-MRI datasets. The constrained CPD algorithm has slightly better mean values for the dice score, however the increase was not significant. The mean source correlation value is clearly better using CPD compared to hNMF. For all the patients, the rank $R$ is selected manually for both constrained CPD and hNMF.

The result of applying the constrained CPD algorithm on one of the MP-MRI datasets from a grade IV patient is 
TABLE I: Dice scores and source correlation of $14 \mathrm{HGG}$ patients using CPD and hNMF algorithms. Dice scores are shown for active tumor and the tumor core (tumor + necrosis) region. Source correlation is shown for active tumor tissue type. Mean and standard deviation are shown in the last two rows, respectively.

\begin{tabular}{|c|c|c|c|c|c|c|}
\hline & \multicolumn{3}{|c|}{ CPD- $l_{1}$} & \multicolumn{3}{c|}{ hNMF } \\
\cline { 2 - 7 } & $\begin{array}{c}\text { Dice } \\
\text { tumor }\end{array}$ & $\begin{array}{c}\text { Dice } \\
\text { core }\end{array}$ & $\begin{array}{c}\text { Tumor source } \\
\text { correlation }\end{array}$ & $\begin{array}{c}\text { Dice } \\
\text { tumor }\end{array}$ & $\begin{array}{c}\text { Dice } \\
\text { core }\end{array}$ & $\begin{array}{c}\text { Tumor source } \\
\text { correlation }\end{array}$ \\
\hline PT01 & 0.83 & 0.96 & 0.94 & 0.81 & 0.98 & 0.50 \\
\hline PT02 & 0.77 & 0.85 & 0.95 & 0.78 & 0.86 & 0.92 \\
\hline PT03 & 0.90 & 0.95 & 0.97 & 0.81 & 0.92 & 0.51 \\
\hline PT04 & 0.81 & 0.88 & 0.95 & 0.74 & 0.82 & 0.88 \\
\hline PT05 & 0.89 & 0.89 & 0.98 & 0.71 & 0.71 & 0.78 \\
\hline PT06 & 0.88 & 0.95 & 0.99 & 0.82 & 0.94 & 0.62 \\
\hline PT07 & 0.76 & 0.88 & 0.80 & 0.74 & 0.90 & 0.54 \\
\hline PT08 & 0.81 & 0.94 & 0.91 & 0.75 & 0.86 & 0.93 \\
\hline PT09 & 0.69 & 0.83 & 0.93 & 0.76 & 0.90 & 0.98 \\
\hline PT10 & 0.79 & 0.59 & 0.95 & 0.54 & 0.50 & 0.74 \\
\hline PT11 & 0.87 & 0.87 & 0.98 & 0.88 & 0.88 & 0.96 \\
\hline PT12 & 0.76 & 0.76 & 0.98 & 0.75 & 0.75 & 0.98 \\
\hline PT13 & 0.92 & 0.92 & 0.98 & 0.93 & 0.93 & 1.00 \\
\hline PT14 & 0.88 & 0.88 & 0.99 & 0.89 & 0.89 & 0.98 \\
\hline Mean & 0.83 & 0.87 & 0.95 & 0.78 & 0.85 & 0.81 \\
\hline std & 0.07 & 0.10 & 0.05 & 0.09 & 0.13 & 0.19 \\
\hline
\end{tabular}

shown in Fig 3 and 4. Fig 3 shows some of the relevant input MRI features (first two rows) and the abundance maps obtained from hNMF and CPD algorithms. For hNMF only four out of six relevant abundance maps are shown, whereas in case of CPD a decomposition of rank $R=4$ was performed and all abundance maps are shown. The sources which represent tissue-specific MP-MRI features obtained from CPD and hNMF algorithms are shown in Fig. 4a. The segmented region of active tumor and necrosis obtained from the CPD and hNMF algorithms is shown in Fig. $4 \mathrm{~b}$ along with the radiologist segmentation. From Fig 3 and 4 we can observe that for this patient (PT03 in Table I) the CPD based algorithm provides a better active tumor segmentation than hNMF based algorithm (Dice tumor ${ }_{C P D}=0.90$ and Dice tumor $_{h N M F}=0.81$ ).

\section{Discussion And Conclusion}

In this paper we have described a method to represent the MP-MRI data in a 3-D tensor. An algorithm using constrained canonical polyadic decomposition with $l_{1}$ regularization was proposed for tissue type differentiation in high-grade glioma patients from multi-parametric MRI. To explore feasibility of the proposed algorithm, it was tested on 14 MP-MRI datasets from patients having high-grade glioma. The constrained CPD algorithm performs slightly better than the existing tissue type differentiation method based on hNMF, but the improvement in dice score is not significant. However, the CPD algorithm has clearly better source correlation than hNMF. Higher source correlation implies that the estimated source features for a particular tissue type better resembles the actual MP-MRI features observed in the region corresponding to that tissue type. This helps in identifying the tissue type from the sources. The main advantage of the tensor approach is that we need to
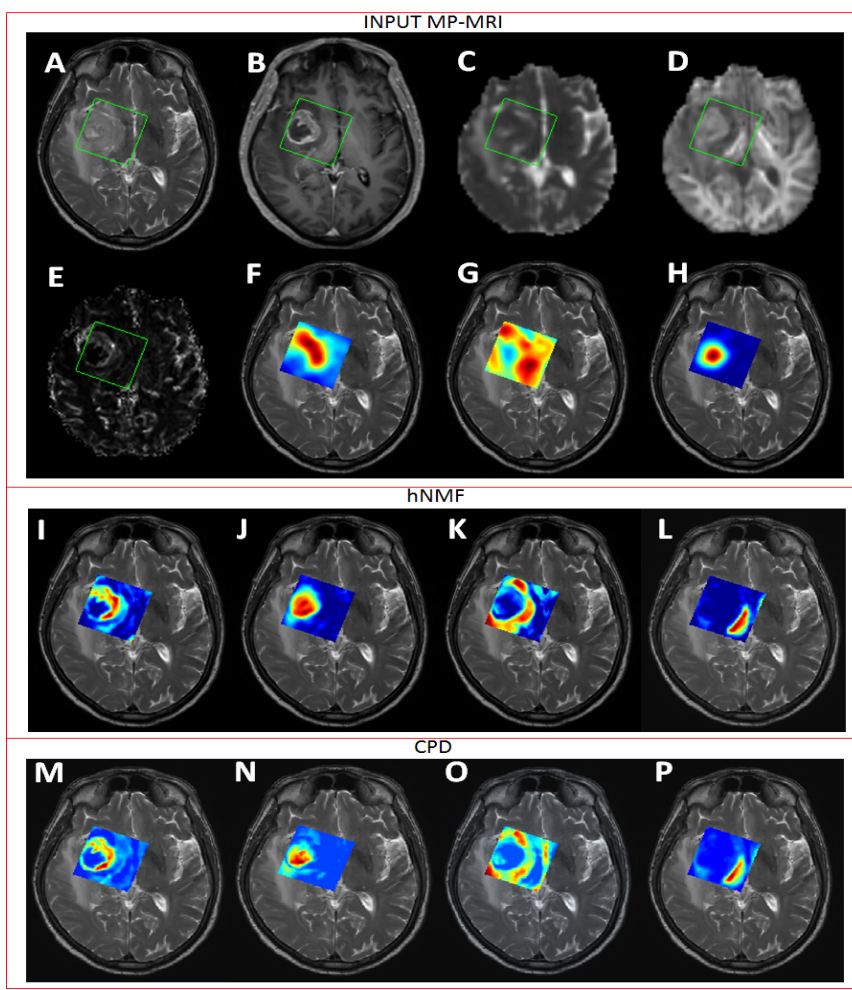

Fig. 3: Coregistered MRI maps of several modalities and tissue abundance maps obtained from hNMF and CPD algorithms. The input maps: (A) T2, (B) T1+C, (C) MD, (D) MK, (E) rCBV, (F) Cho, (G) Cre and (H) Lip. The green box indicates the ROI. (I-L) tissue abundance maps obtained from hNMF ((I) Active tumor, (J) Necrosis, (K) Edema). (M-P) tissue abundance maps obtained from constrained CPD ((M) Active tumor, (N) Necrosis, (O)) Edema).

select only one rank $R$, whereas in the hNMF algorithm we need to select two ranks, one for each group in the second step of hNMF [5]. Therefore, automation of the CPD based algorithm will be much easier compared to hNMF. Also in the tensor approach, the tensor construction couples different MP-MRI features because of the $X X^{T}$ in the frontal slices. Therefore, in the sources obtained from the CPD algorithm the features will be coupled, meaning that an individual feature will not appear as a separate source. This is the reason for having higher source correlation values in the CPD algorithm. Moreover, for each voxel, the $X X^{T}$ in the frontal slices will give more weight to dominant features and least significant features will be suppressed. In this in-vivo study we have shown that a tensor formulation can be used for tumor characterization in multi-parametric MRI and further research in this direction is promising.

\section{ACKNOWLEDGMENT}

The authors would like to thank the University Hospitals of Leuven and in particular radiologist Dr. Sofie Van Cauter for data acquisition and segmenting the pathological tissue. This research was supported by: Flemish Government FWO project G.0869.12N (Tumor imaging), G.0830.14N 
A

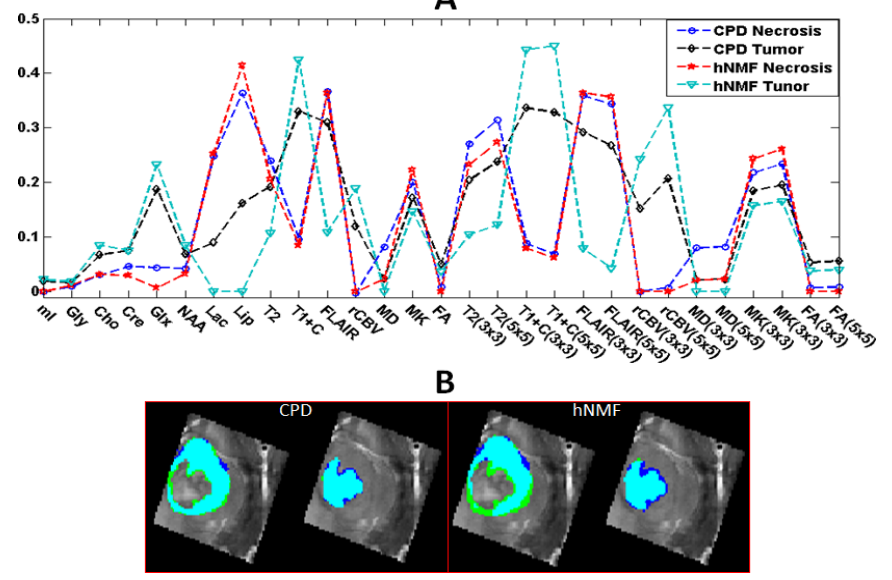

Fig. 4: (A) Sources consisting of MP-MRI features obtained from CPD and hNMF algorithms. The estimeted sources are shown for tumor and necrosis tissue type. (B) Comparison of the segmentation by radiologist (blue) with the segmentations obtained from CPD and hNMF (green). First two images correspond to tumor and necrosis segmentation from CPD, respectively. Last two images correspond segmentations from hNMF. Cyan indicates segmentation overlap.

(Block term decompositions); IWT IM 135005; Belgian Federal Science Policy Office: IUAP P7/19 (DYSCO, 'Dynamical systems, control and optimization', 2012-2017); Research Council KUL: CoE PFV/10/002 (OPTEC); EU: European Research Council under the European Union's Seventh Framework Programme (FP7/2007-2013): EU MC ITN TRANSACT 2012, \#316679 and ERC Advanced Grant, \#339804 BIOTENSORS. This paper reflects only the authors views and the Union is not liable for any use that may be made of the contained information.

\section{REFERENCES}

[1] Y. Li, D. M. Sima, S. V. Cauter, Croitor Sava A. R., U. Himmelreich, Y. Pi, and S. Van Huffel, "Hierarchical non-negative matrix factorization (hNMF): a tissue pattern differentiation method for glioblastoma multiforme diagnosis using MRSI," NMR in Biomedicine, vol. 26, no. 3, pp. 307-319, 2013.

[2] B. P. Keogh and J. W. Henson, "Clinical manifestations and diagnostic imaging of brain tumors," Hematology/oncology clinics of North America, vol. 26, no. 4, pp. 733-755, 2012.

[3] S. Dimou, R. Battisti, D. Hermens, and J. Lagopoulos, "A systematic review of functional magnetic resonance imaging and diffusion tensor imaging modalities used in presurgical planning of brain tumour resection," Neurosurgical review, vol. 36, no. 2, pp. 205-214, 2013.

[4] S. Van Cauter, F. De Keyzer, D. M. Sima, Croitor Sava A. R., F. D'Arco, J. Veraart, R. R. Peeters, A. Leemans, S. Van Gool, G. Wilms, P. Demaerel, S. Van Huffel, S. Sunaert, and U. Himmelreich, "Integrating diffusion kurtosis imaging, dynamic susceptibilityweighted contrast-enhanced MRI, and short echo time chemical shift imaging for grading gliomas," Neuro-oncology, vol. 16, no. 7, pp. 1010-1021, 2014.

[5] N. Sauwen, D. M. Sima, S. Van Cauter, J. Veraart, A. Leemans, F. Maes, U. Himmelreich, and S. Van Huffel, "Hierarchical nonnegative matrix factorization to characterize brain tumor heterogeneity using multi-parametric MRI," NMR in Biomedicine, vol. 28, no. 12, pp. 1599-1624, 2015.

[6] O. Debals, M. Van Barel, and L. De Lathauwer, "Lowner-based blind signal separation of rational functions with applications," Signal Processing, IEEE Transactions on, vol. PP, no. 99, pp. 1-1, 2015.
[7] H. N. Bharath, D. M. Sima, N. Sauwen, U. Himmelreich, L. De Lathauwer, and S. Van Huffel, "Tensor based tumor tissue type differentiation using magnetic resonance spectroscopic imaging," in Engineering in Medicine and Biology Society (EMBC), 2015 37th Annual International Conference of the IEEE, Aug 2015, pp. 7003-7006.

[8] D. N. Louis, H. Ohgaki, O. D. Wiestler, W. K. Cavenee, P. C. Burger, A. Jouvet, B. W. Scheithauer, and P. Kleihues, "The 2007 WHO classification of tumours of the central nervous system," Acta neuropathologica, vol. 114, no. 2, pp. 97-109, 2007.

[9] "Dynamic Susceptibility Contrast MR ANalysis (DSCoMAN)," Available at: https://sites.duke.edu/dblab/dscoman/, accessed: 2016-02-01.

[10] C. A. Schneider, W. S. Rasband, K. W. Eliceiri et al., "NIH Image to ImageJ: 25 years of image analysis," Nat methods, vol. 9, no. 7, pp. 671-675, 2012.

[11] J. Boxerman, K. Schmainda, and R. Weisskoff, "Relative cerebral blood volume maps corrected for contrast agent extravasation significantly correlate with glioma tumor grade, whereas uncorrected maps do not," American Journal of Neuroradiology, vol. 27, no. 4, pp. 859867, 2006.

[12] S. Van Cauter, D. M. Sima, J. Luts, L. ter Beek, A. Ribbens, R. R. Peeters, M. I. Osorio Garcia, Y. Li, S. Sunaert, S. W. Van Gool et al., "Reproducibility of rapid short echo time CSI at 3 tesla for clinical applications," Journal of Magnetic Resonance Imaging, vol. 37, no. 2, pp. 445-456, 2013.

[13] R. J. Ogg, R. Kingsley, and J. S. Taylor, "WET, a T1-and B1insensitive water-suppression method for in vivo localized ${ }^{1} \mathrm{H}$ NMR spectroscopy," Journal of Magnetic Resonance, Series B, vol. 104, no. 1, pp. 1-10, 1994.

[14] J. B. Poullet, "Quantification and classification of magnetic resonance spectroscopic data for brain tumor diagnosis," Ph.D. dissertation, PhD Thesis. Leuven, Belgium, 2008.

[15] Croitor Sava A. R., D. M. Sima, J.-B. Poullet, A. J. Wright, A. Heerschap, and S. Van Huffel, "Exploiting spatial information to estimate metabolite levels in two-dimensional MRSI of heterogeneous brain lesions," NMR in Biomedicine, vol. 24, no. 7, pp. 824-835, 2011.

[16] J.-B. Poullet, D. M. Sima, S. Van Huffel, and P. Van Hecke, "Frequency-selective quantitation of short-echo time ${ }^{1} \mathrm{H}$ magnetic resonance spectra," Journal of Magnetic Resonance, vol. 186, no. 2, pp. 293-304, 2007.

[17] D. H. Poot, A. J. den Dekker, E. Achten, M. Verhoye, and J. Sijbers, "Optimal experimental design for diffusion kurtosis imaging," Medical Imaging, IEEE Transactions on, vol. 29, no. 3, pp. 819-829, 2010.

[18] J. Andersson, J. Xu, E. Yacoub, E. Auerbach, S. Moeller, and K. Ugurbil, "A comprehensive gaussian process framework for correcting distortions and movements in diffusion images," in Proceedings of the 20th Annual Meeting of ISMRM, 2012, p. 2426.

[19] J. Veraart, J. Sijbers, S. Sunaert, A. Leemans, and B. Jeurissen, "Weighted linear least squares estimation of diffusion MRI parameters: strengths, limitations, and pitfalls," NeuroImage, vol. 81, pp. 335-346, 2013.

[20] D. Le Bihan, J.-F. Mangin, C. Poupon, C. A. Clark, S. Pappata, N. Molko, and H. Chabriat, "Diffusion tensor imaging: concepts and applications," Journal of magnetic resonance imaging, vol. 13, no. 4, pp. 534-546, 2001.

[21] "Statistical Parametric Mapping (SPM)," Available at: http://www.fil.ion.ucl.ac.uk/spm/, accessed: 2016-02-01.

[22] F. Maes, A. Collignon, D. Vandermeulen, G. Marchal, and P. Suetens, "Multimodality image registration by maximization of mutual information," Medical Imaging, IEEE Transactions on, vol. 16, no. 2, pp. 187-198, 1997.

[23] A. Leemans, B. Jeurissen, J. Sijbers, and D. Jones, "ExploreDTI: a graphical toolbox for processing, analyzing, and visualizing diffusion MR data," in 17th Annual Meeting of Intl Soc Mag Reson Med, vol. 209, 2009, p. 3537.

[24] A. Cichocki, D. Mandic, L. De Lathauwer, G. Zhou, Q. Zhao, C. Caiafa, and H. Phan, "Tensor decompositions for signal processing applications: From two-way to multiway component analysis," Signal Processing Magazine, IEEE, vol. 32, no. 2, pp. 145-163, March 2015.

[25] L. Sorber, V. Barel, and L. D. Lathauwer, "Tensorlab v2.0," Available online, January 2014.

[26] L. Sorber, M. Van Barel, and L. De Lathauwer, "Structured data fusion," Selected Topics in Signal Processing, IEEE Journal of, vol. 9, no. 4, pp. 586-600, June 2015. 